\title{
Spectroscopic Signatures of MQ-Resins in Silicone
}

\section{Elastomers}

April M. Sawvel', Jonathan C. Crowhurst ${ }^{1}$, Harris E. Mason², James S. Oakdale',

Samantha Ruelas ${ }^{1}$, Hannah V. Eshelman ${ }^{1}$, and Robert S. Maxwel/,1.

${ }^{1}$ Physical and Life Sciences Directorate, Materials Science Division, Lawrence

Livermore National Laboratory, 7000 East Avenue, Livermore, CA 94550.

${ }^{2}$ Physical and Life Sciences Directorate, Atmospheric, Earth, and Energy Division,

Lawrence Livermore National Laboratory, 7000 East Avenue, Livermore, CA 94550.

SUPPORTING INFORMATION 


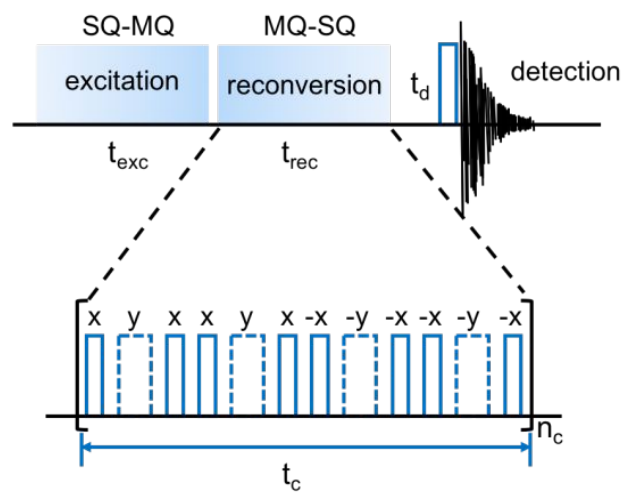

Figure $\mathrm{S} 1 .{ }^{1} \mathrm{H} \mathrm{MQ}$ pulse sequence used in this study. Solid lines represent $\pi / 2$ pulses and dashed lines represent $\pi$ pulses. The number of pulse trains $\left(n_{\mathrm{c}}\right)$ was incremented to generate growth curves. Reference $\mathrm{MQ}$ coherences, containing all excited $4 n$ quantum orders, were selected using the $\mathrm{DQ}$ (double quantum) selection phase cycle without receiver alternation.

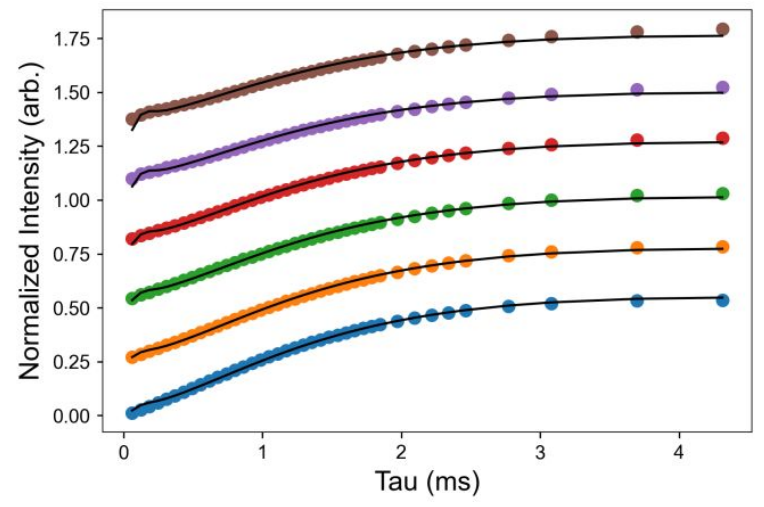


Figure S2. Normalized DQ build-up curves and fits produced using our hybrid fitting method. The curves start with the $0 \%$ MQ sample at the bottom (blue) and then go through the series of samples from 10 to 50\% MQ. The curves have been offset in this Figure for clarity.

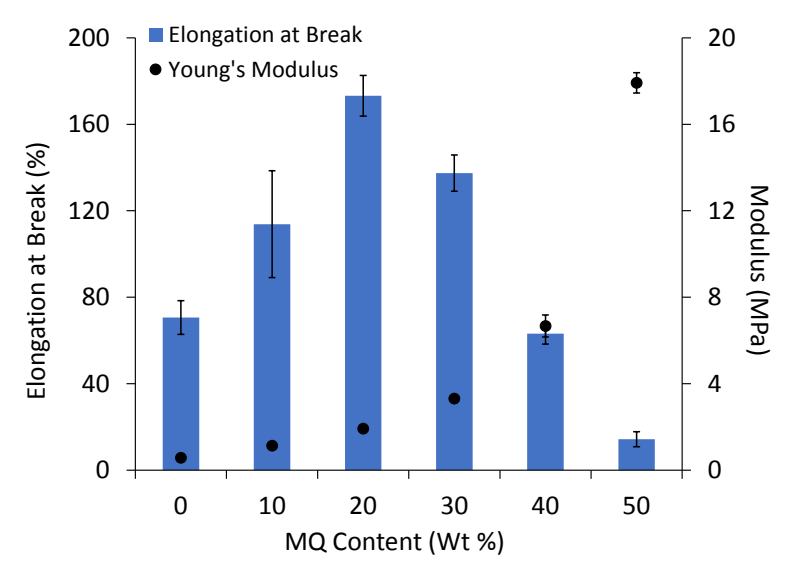

Figure S3. Young's modulus (MPa) and elongation at break (\%) plotted as a function of MQ-resin concentration in our model network samples. A sharp decline in the elongation at break value above 30\% MQ-resin content corresponds with the observed sharp increase in the Young's modulus value of these samples. 


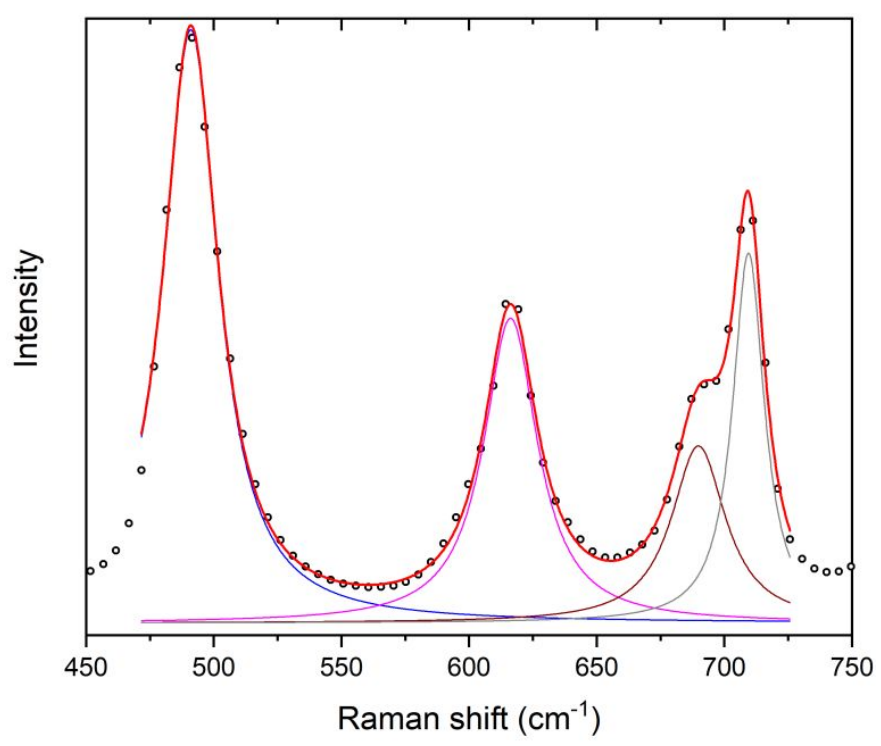

Figure S4. The region of the Raman spectrum of the 50\% MQ sample relevant to the calibration curve shown in the main text. A sum of Lorentzian functions was fitted to the spectrum after a linear background subtraction. The various components are indicated as well as their cumulative line shape.

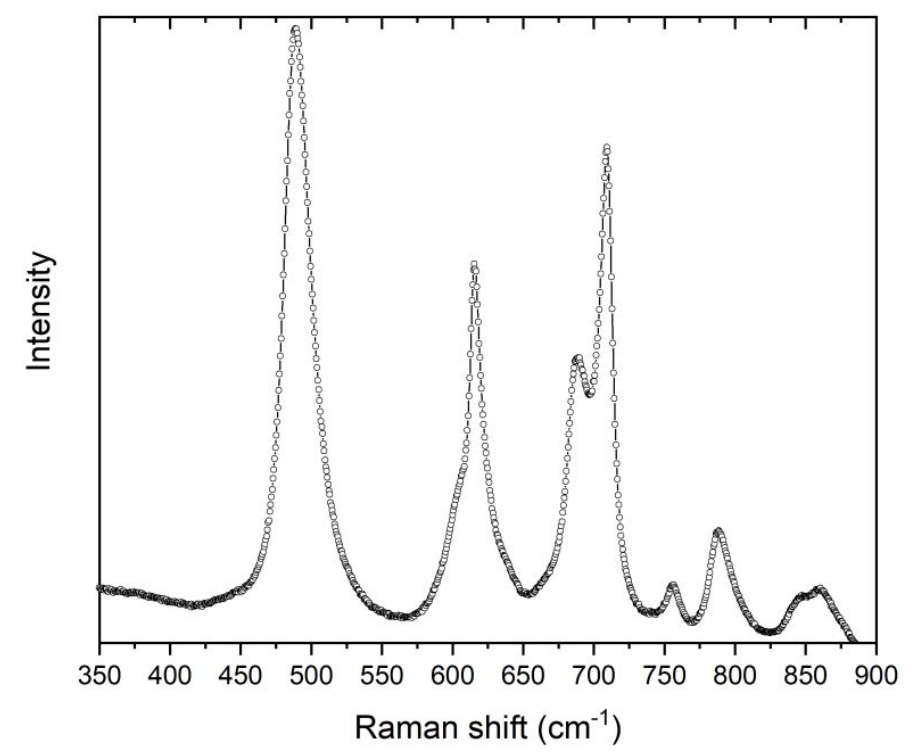


Figure S5. High resolution Raman spectrum of the 50\% MQ sample. The spectral region covers the same range as that of Figure 2 of the main text.

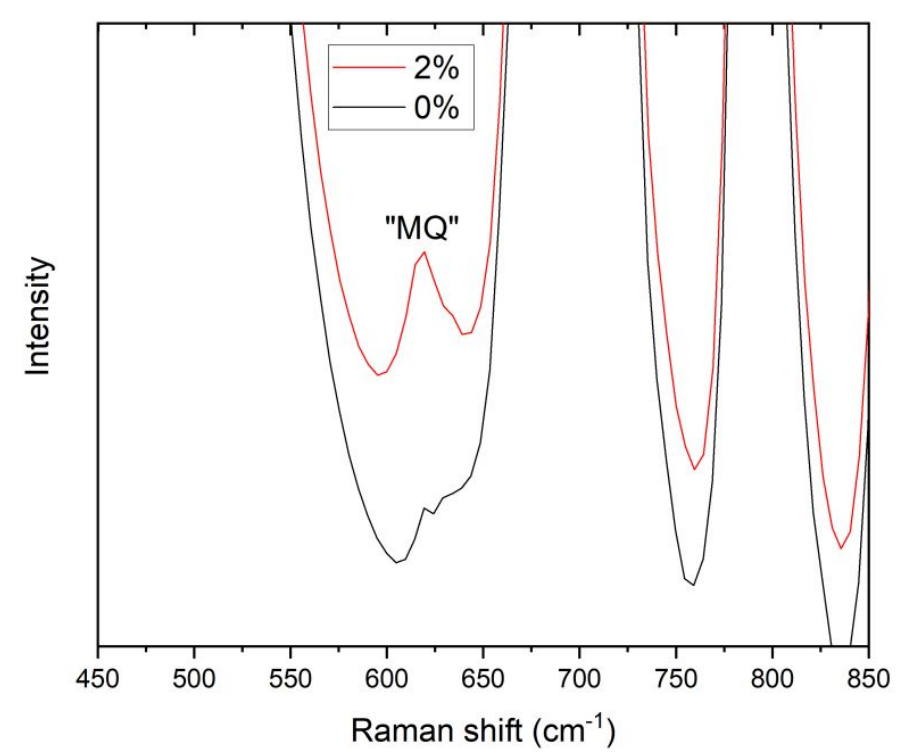

Figure S6. Raman spectra of the $0 \%$ MQ sample compared to the $2 \%$ MQ sample. Spectra normalized to the Si-O-Si stretch of PDMS. There is activity in the MQ region even in the $0 \%$ MQ sample, possibly due to MQ-like moieties created from condensed cross-linker. 


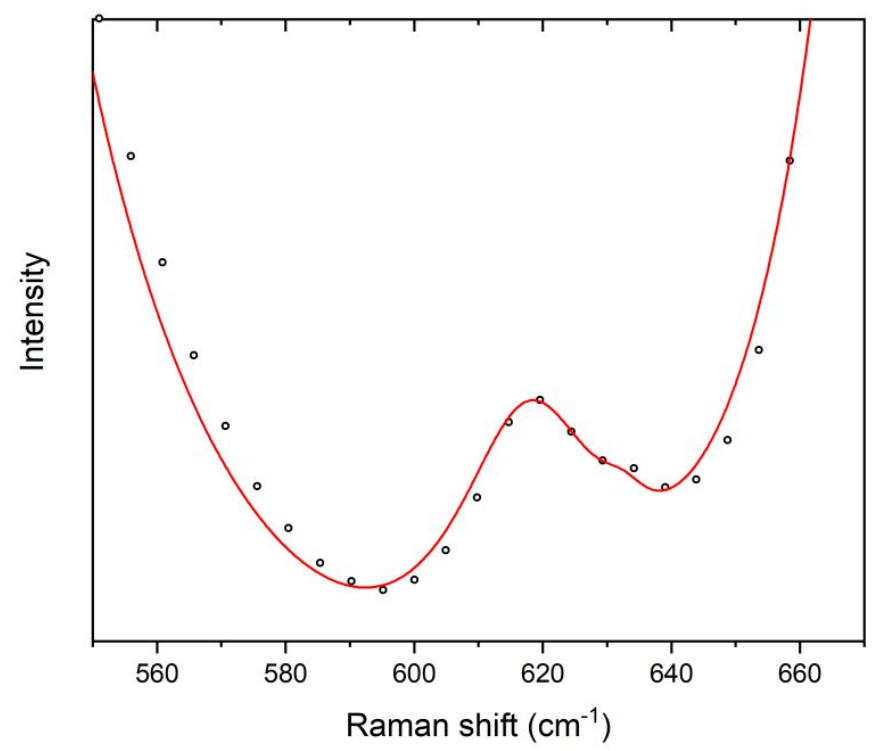

Figure S7. Magnified Raman spectrum of the $2 \%$ MQ sample. Solid line is a fit to the spectrum. Five Lorentzian components were used (instead of 4 as in Figure S2) to capture the weak contribution from the native material in the MQ spectral region.
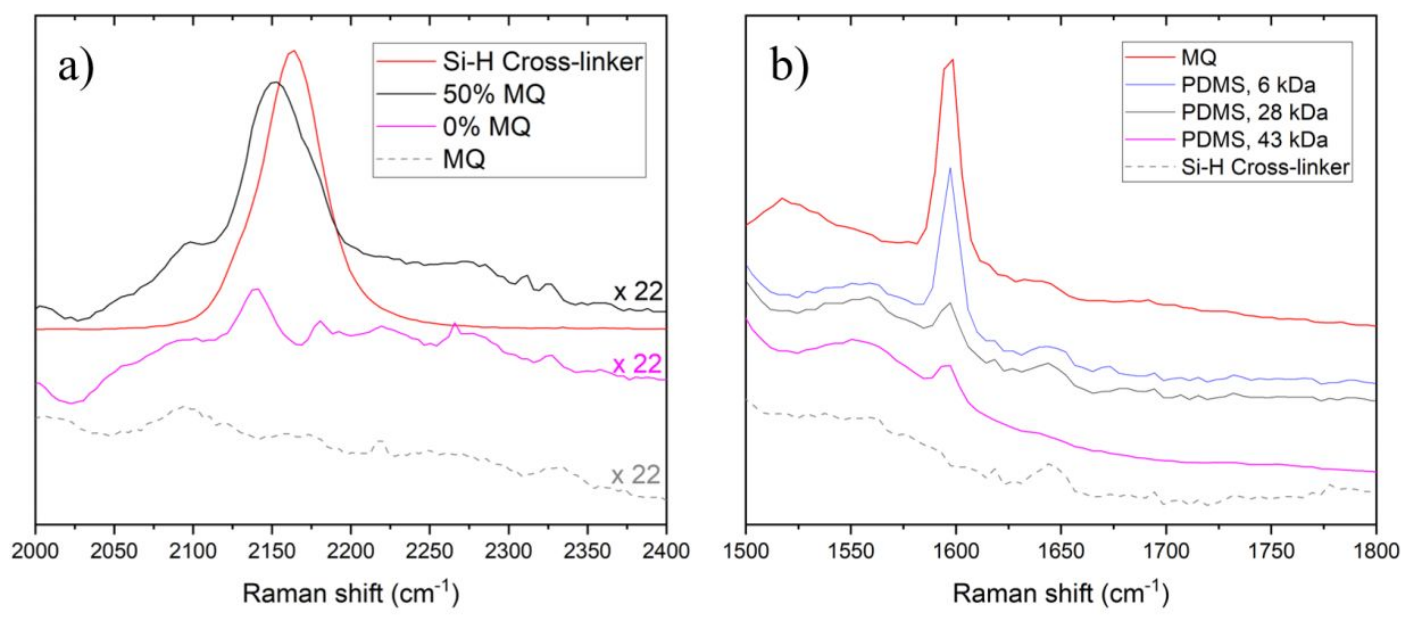

Figure S8. Raman spectra of the a) Si-H stretch region for the cross-linker (HMS-H271), the $50 \% \mathrm{MQ}$ sample, the $0 \% \mathrm{MQ}$ sample, and pure MQ-resin. b) Raman spectra of the $\mathrm{C}=\mathrm{C}$ stretch region for pure MQ-resin, the cross-linker (HMS-H271), and three different molecular weight 
PDMS chains $(\mathrm{DMS}-\mathrm{V} 21=6 \mathrm{kDa}$, DMS-V31 $=28 \mathrm{kDa}$, and DMS-V33 $=48 \mathrm{kDa})$. Raman spectra have been scaled and shifted vertically to permit comparison.

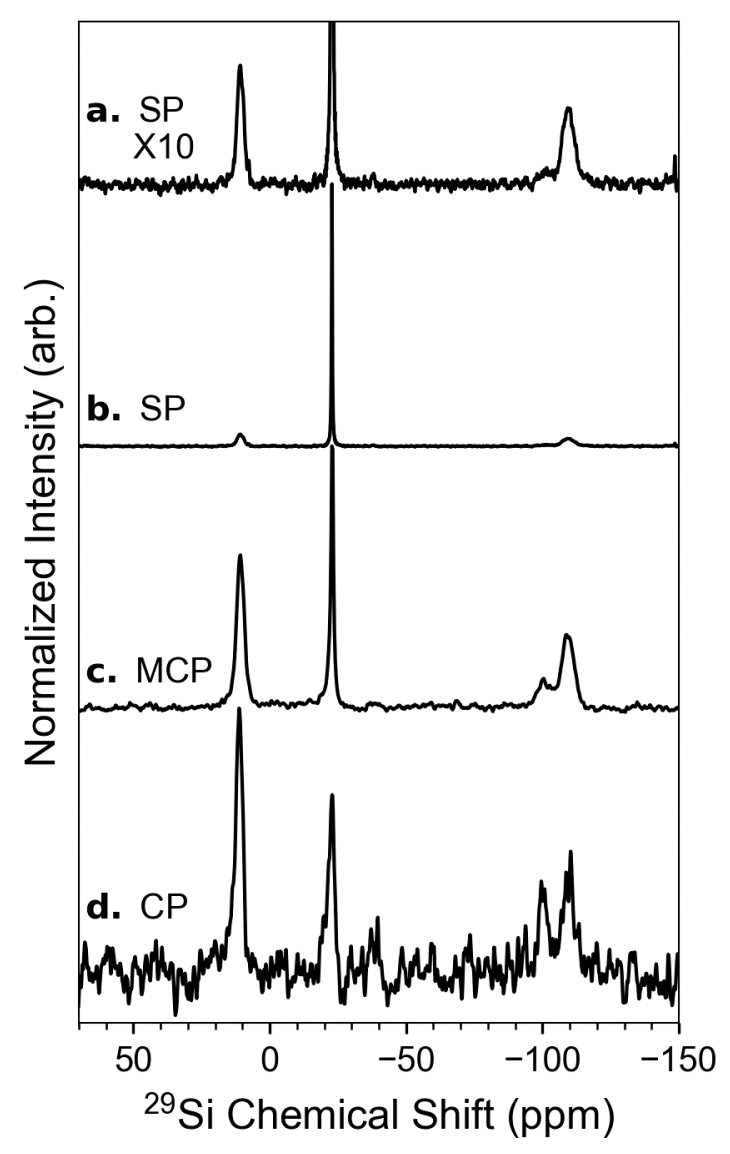

Figure S9. Comparison of a-b) $\left.{ }^{29} \mathrm{Si} \mathrm{SP} / \mathrm{MAS}, \mathrm{c}\right){ }^{29} \mathrm{Si}\left\{{ }^{1} \mathrm{H}\right\}$ MultiCP, and d) traditional ${ }^{29} \mathrm{Si}\left\{{ }^{1} \mathrm{H}\right\}$ CP/MAS experiments collected on the 50\% MQ Resin sample. 


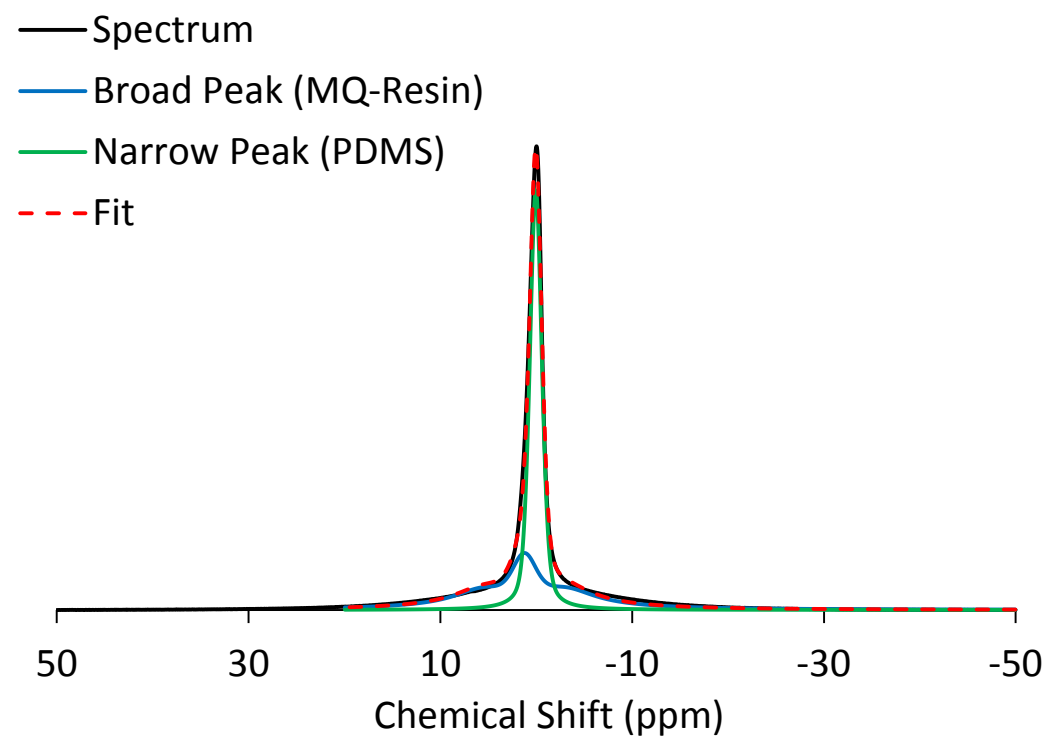

Figure S10. Static ${ }^{1} \mathrm{H}$ NMR spectra of the $50 \%$ MQ sample showing the broad spectral component that increases in intensity with MQ content. 


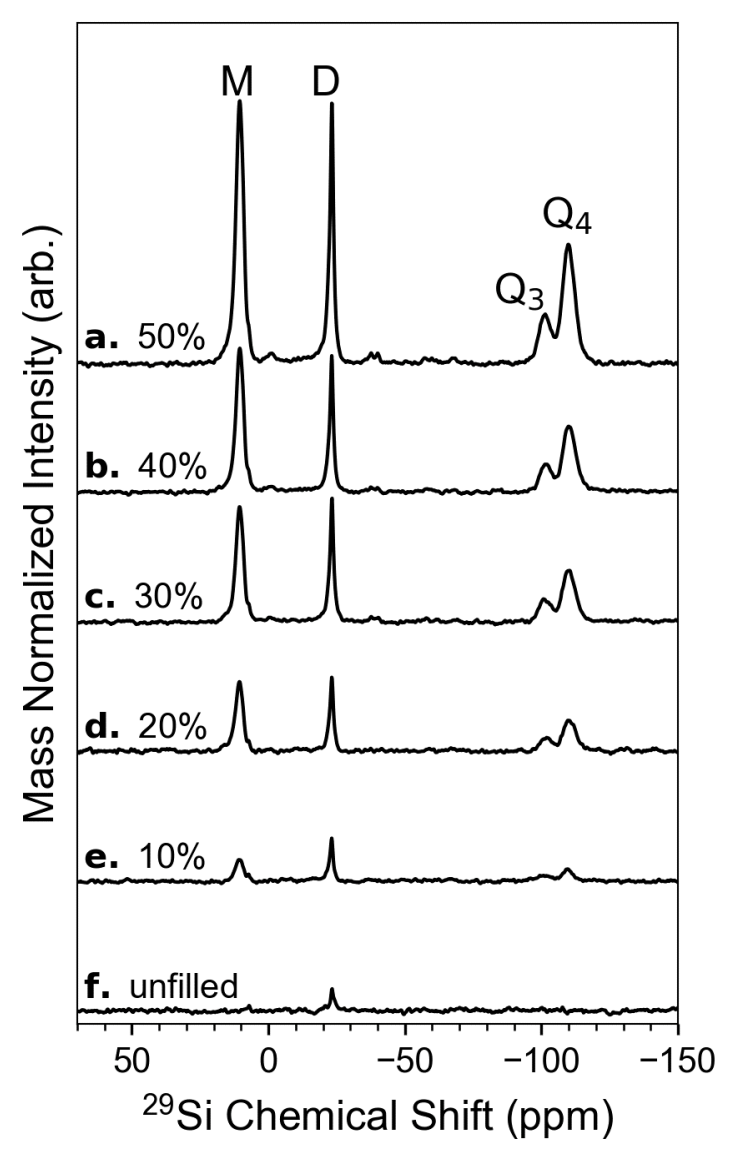

Figure S11. "Traditional" ${ }^{29} \mathrm{Si}\left\{{ }^{1} \mathrm{H}\right\} \mathrm{CP} / \mathrm{MAS}$ spectra collected using a $10 \mathrm{~ms}$ contact pulse for the MQ samples in descending order of MQ content. The intensities have been scaled relative to the number of scans and normalized to the sample mass. 


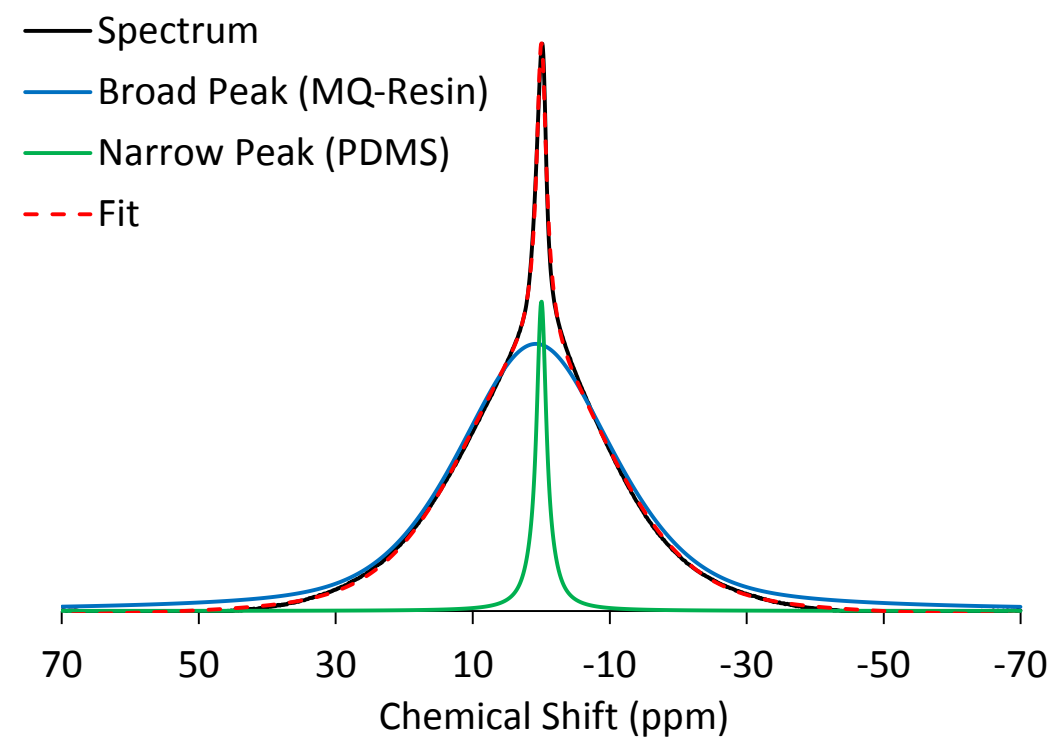

Figure S12. An example of the ${ }^{1} \mathrm{H}$ DQ NMR spectrum for the first DQ excitation time $(61.6 \mu \mathrm{s})$ of the 50\% MQ sample. The broad underlying component increases in intensity with MQ content and has been assigned to the high ${ }^{1} \mathrm{H}_{-}{ }^{1} \mathrm{H}$ dipolar coupling interactions in the MQ-resin. 